\title{
Association of the Patient-Centered Medical Home Implementation with Chronic Disease Quality in Patients with Multimorbidity
}

\author{
Linnaea Schuttner, $M D, M S^{1,2}$ (D) Edwin S. Wong, $P h D^{1,3}$, Ann-Marie Rosland, $M D, M S^{4,5}$, \\ Karin Nelson, MD, MSHS ${ }^{1,2}$, and Ashok Reddy, MD, $\mathrm{MS}^{1,2}$
}

${ }^{1}$ Health Services Research \& Development, VA Puget Sound Health Care System, 1660 South Columbian Way, Seattle, WA, USA; ${ }^{2}$ Department of Medicine, University of Washington School of Medicine, Seattle, WA, USA; ${ }^{3}$ Department of Health Services, University of Washington School of Public Health, Seattle, WA, USA; “VA Center for Health Equity Research and Promotion, VA Pittsburgh Health Care System, Pittsburgh, PA, USA;

${ }^{5}$ Department of Internal Medicine, University of Pittsburgh School of Medicine, Pittsburgh, PA, USA.

BACKGROUND: The patient-centered medical home (PCMH) was established in part to improve chronic disease management, yet evidence is limited for effects on patients with multimorbidity.

OBJECTIVE: To examine the association of PatientAligned Care Team (PACT) implementation, the Veterans Health Administration (VA) PCMH model, and care quality for multimorbid patients enrolled in VA primary care from 2012 to 2014.

DESIGN: Retrospective cohort.

PATIENTS: 318,764 multimorbid ( $>3$ chronic diseases) patients receiving care in 917 clinics.

MAIN MEASURES: PCMH implementation was measured using the PACT Implementation Progress Index $\left(\mathrm{PI}^{2}\right)$ for clinics in 2012. The $\mathrm{PI}^{2}$ is a validated composite measure of administrative and survey data with higher scores associated with greater care quality. Quality outcomes from 2013 to 2014 were assessed from External Peer Review Program (EPRP) metrics. Outcomes included preventative care, chronic disease management, and mental health and substance use metrics. We used generalized estimating equations to model associations adjusting for patient and clinic characteristics. We also examined associations for a subgroup with $>5$ chronic diseases.

KEY RESULTS: For one-third of metrics (5/15), greater implementation of PACT in 2012 was associated with higher predicted probability of meeting the quality metric in 2013-2014. This association persisted for only two metrics (diabetic glycemic control, $P<0.001$; lipid control in ischemic heart disease, $P=0.02$ ) among patients with $\geq$ 5 chronic diseases.

CONCLUSIONS: Multimorbid patients engaged in care from clinics with higher PCMH implementation received higher quality care across several quality domains, but this association was reduced in patients with $\geq 5$ chronic diseases.

Electronic supplementary material The online version of this article (https://doi.org/10.1007/s11606-020-06076-7) contains supplementary material, which is available to authorized users.

Previous Presentation This work was previously presented at the Society for General Internal Medicine Annual Conference, May 8-11, 2019, Washington, D.C., and the Academy Health Annual Research Meeting, June 4-6, 2019, Washington, D.C.

Received January 9, 2020

Accepted July 17, 2020

Published online August 6, 2020
KEY WORDS: quality improvement; primary care; comorbidity; patientcentered medical homes; chronic disease management

J Gen Intern Med 35(10):2932-8 DOI: $10.1007 / \mathrm{s} 11606-020-06076-7$

(c) Society of General Internal Medicine (This is a U.S. government work and not under copyright protection in the U.S.; foreign copyright protection may apply) 2020

\section{INTRODUCTION}

Patients with multiple chronic diseases, or multimorbidity, comprise over half of those age $\geq 65 .{ }^{1,2}$ Multimorbid patients are a vulnerable population, with worse health outcomes and higher mortality. ${ }^{3-5}$ Compared with those with fewer diagnoses, they interact with health systems differently. They have more face-toface primary care visits - creating opportunities for screenings and disease monitoring. ${ }^{6,7}$ At the same time, they have greater medical complexity and socioeconomic needs, which can lead to challenges for primary care providers (PCPs) balancing competing demands with chronic care needs. ${ }^{2,}, 8,9$

The patient-centered medical home (PCMH) is based on the chronic care model, which provides a framework for understanding the care of chronic diseases..$^{10}$ Despite relevance to multimorbid patients, effects of the PCMH for these patients are uncertain. In 2010, the Veterans Health Administration (VA) began implementing a PCMH model, the PatientAligned Care Team (PACT) initiative, in over 900 primary care clinics nationwide. The PACT model focused on chronic disease management, expanded access and continuity, comprehensive care through population-based screenings and disease registries, and patient-centeredness through staff training and care redesign. ${ }^{11}$ Patients within PACT are assigned to "teamlets" with a core of a primary care provider (PCP) and nurse care manager. Teamlets belong to larger teams with interdisciplinary support staff, including integrated behavioral health. Despite organizational similarity, PACT was not implemented uniformly at all sites. In observational studies, clinics with greater PACT implementation delivered better quality care, had lower preventable hospitalization rates for patients, and higher patient satisfaction. ${ }^{12,13}$ 
Despite promising findings within the VA overall, the potential impact of PACT on multimorbid patients is less clear. Within PACT, increased attention on chronic disease management could result in conflicting therapies (e.g., NSAIDs with osteoarthritis and hypertension) or unsustainable drug regimens for patients eligible for multiple disease guidelines. ${ }^{14}$ ${ }^{15}$ Alternatively, comprehensive screenings could have widespread ripple effects, such as the consequences of detecting and treating depression for patient engagement in care. ${ }^{16}$ Understanding the effect of PACT implementation specifically for multimorbid patients could inform resource distribution and identify gaps in care processes critical for this highrisk group. To address this, we examined quality metrics for preventative care and chronic disease management across a national sample of multimorbid patients receiving care from the VA.

\section{METHODS}

\section{Study Overview and Data Sources}

We performed a retrospective cohort study examining the association of a clinic-level measure of PACT implementation in 2012 with quality outcomes in 2013-2014. We selected these years to fall after the national rollout of PACT, yet prior to community care expansion in the Choice Act of 2014. ${ }^{17}$ Quality outcomes were from the VA's External Peer Review Program (EPRP) database. Provider and clinic data were from the VA Provider Specialty Workforce Report and VA Site Tracking System. Patient characteristics and utilization were from the VA Corporate Data Warehouse. ${ }^{18}$ Rural status was per the US Census Bureau. This study was part of the VA's PACT evaluation and considered quality improvement rather than research, and was therefore not subject to institutional review board approval or waiver.

\section{Cohort Description}

We identified 450,019 Veterans enrolled in the VA in fiscal year (FY) 2012 (10/1/11-9/30/12), with a quality metric measured in late FY2013 through FY2014 (4/1/13-10/30/14). Patients were excluded if they were not seen in a primary care clinic in $2012(n=13,091,2.9 \%)$, were missing covariates $(n=4850,1.1 \%)$, or were not multimorbid $(n=112,847$, $25.1 \%$ ). Multimorbidity was defined as $\geq 3$ chronic diseases in $\geq 3$ body systems based on encounter diagnosis codes during FY2012. Diseases and body systems were categorized using ICD-9 codes according to the Agency for Healthcare Research and Quality's Chronic Condition Index.., ${ }^{19}$ Our final cohort included 318,764 patients.

\section{Quality Outcomes}

Previous observational studies have examined the relationship between PACT implementation and quality metrics for 48 available EPRP metrics relating to chronic disease management, mental health and substance use disorder screening, and preventative care. ${ }^{13,}{ }^{20}$ We drew from conceptual frameworks to determine which metrics were chosen and develop our understanding of complexity within multimorbid patients. ${ }^{8-10}$ From this list of 48 metrics, we selected 16 metrics a priori that best represented how the medical home might affect quality metrics for multimorbid patients and/or be mediated by patient complexity through the domains of the $\mathrm{PCMH}$, such as increased screenings through team-based care and chronic disease management, and/or be mediated by patient complexity, such as potential clinical tradeoffs in care needs. We excluded metrics that could be potentially inappropriate for patients with reduced life expectancy or increased disease burden (such as malignancy screening) and metrics related to actions not directly performed by the PACT team (such as teleretinopathy screening for diabetics). We selected 16 metrics for high-prevalence chronic diseases in the VA (diabetes, cardiovascular disease, mental health, substance use disorders), ${ }^{21}$ and vaccines to represent non-disease or gender-specific preventative care processes broadly applicable to multimorbid patients. EPRP metrics are analogous to other national quality performance metrics used outside the VA (such as HEDIS measures). They are abstracted from charts by an independent group from a stratified random sample of outpatients seen in the past month, with oversampling among patients with chronic conditions. ${ }^{22}$ Patients are eligible independently for each metric and can be sampled once per metric per year. If selected more than 1 time for the same metric during the study period (as this spanned across 2 years), we used the first measurement. For this analysis, patients were permitted to be part of multiple denominators as outcomes were assessed independently.

\section{PACT Implementation}

Our primary independent variable was the level of PACT implementation at a patient's primary care clinic in 2012. The level of PACT implementation was measured using the previously validated $\mathrm{PI}^{2}$ score, a composite of administrative and survey data capturing 8 PCMH domains: access; continuity; care coordination; comprehensiveness; self-management support; patient-centered care; shared decision-making; and team functioning. ${ }^{13}$ The extent to which clinics implemented each domain was measured with standardized $z$-scores. Total clinic scores were calculated as the number of domains within the top versus bottom quartiles, ranging from -8 (all domains in bottom quartile) to +8 (all domains in top quartile). We employed previous divisions for categories of PACT implementation ranging from low to high levels of implementation ( -8 to $-5 ;-4$ to $-2 ;-1$ to $1 ; 2$ to $4 ; 5$ to 8$).{ }^{13,}{ }^{20}$ Previous observational studies have shown that clinics measuring in the highest quartile for all domains had higher patient satisfaction with care by a full 1 point (on a 10 point scale), lower staff burnout by 0.5 points on the Maslach Burnout Inventory, 57 fewer emergency department visits and 6 fewer 
hospitalizations per 1000 patients compared with those clinics in the bottom quartile for all domains. ${ }^{13}$

\section{Covariates}

We adjusted for potential confounders from 2012 consistent with prior studies. ${ }^{13,20}$ These included patient characteristics (age, sex, race/ethnicity, copay exemption, marital status, median household income by residence county, travel distance to clinic), and clinic characteristics (full time equivalent PCPs per 10,000 patients, location (urban vs. rural), hospital or community affiliation), and a time variable for quarter of sampling year.

\section{Statistical Analyses}

We performed descriptive analyses separately to characterize the overall cohort and patients within each metric. In these analyses, we compared characteristics of patients receiving care at the top versus bottom quintile of PACT implementation clinics using unequal variance $t$ tests or Pearson's chi-square tests as appropriate. We used generalized estimating equations (GEE) with a logit link to estimate associations between $\mathrm{PI}^{2}$ quintile and dichotomous quality metrics, adjusting for covariates. We assumed an exchangeable correlation working structure to accommodate increased correspondence of outcomes within the same clinic. We converted coefficients to predicted probabilities assuming unbalanced data. We used ANOVA for tests of trend. Standard errors were heteroskedastic robust with two-sided hypothesis testing and an alpha of 0.05 . Analyses were conducted using $\mathrm{R}$ 3.5.0 (www.r-project.org) and Stata 14.0 (StataCorp, 2015, College Station, TX).

We performed several secondary analyses. First, as an effect modifier, we examined the interaction of prior hospitalizations in 2012 with PACT implementation. We selected hospitalizations as a marker of illness severity that could potentially indicate differential care receipt (for example, acute illnesses that would be prioritized over vaccination). ${ }^{23}$ Next, we examined the interaction of total primary care visits during 2012 and PACT implementation to determine if the "dose" of PACT exposure was important. If the interaction term for the effect modifier and the predictor was significant using ANOVA, we then conducted joint tests of the predicted probability of outcomes between groups differing across levels of effect modifier (that is, comparing patients with and without prior hospitalizations, across a clinic $\mathrm{PI}^{2}$ category) to assess effect size differences. In addition to these effect modifier analyses, we performed a sensitivity analyses using only those clinics with a minimum of $\geq 5$ patients to assess for influence of statistical "noise" from low volumes. Finally, to approximate effects for complexity, we examined effects for patients with $>5$ chronic diseases in $\geq 5$ body systems $(n=180,672) .{ }^{24-26}$

\section{RESULTS}

Patients with multimorbidity were the majority (70\%) of those engaged in care in 2012 who also had quality metrics available in 2013-2014 (Fig. 1). Patients receiving care from top performing clinics for PACT implementation were older $(65$ vs. 64 years), more male ( $88 \%$ vs. $86 \%$ ), non-Hispanic White $(80 \%$ vs. $68 \%)$, and seen more in rural $(41 \%$ vs. $25 \%)$ and community clinics (63\% vs. $51 \%)$, compared with patients in the lowest performing clinics (all $P<0.001$, Table 1).

After covariate adjustment, there was a significant trend for 5 of 15 quality metrics between greater implementation of PACT and higher probability of achieving the quality metric. These included glycemic control (trend, $P<0.001$ ) and lipid control $(P=0.04)$ among diabetics; lipid control $(P<0.01)$ among ischemic heart disease (IHD) patients; influenza vaccination $\geq 65$ years $(P=0.02)$; and timely follow-up of positive post-traumatic stress disorder (PTSD) screening $(P=$ 0.02 , Table 2 ). The overall difference in probability of meeting quality metrics between clinics with lowest to highest PACT implementation ranged from 2\% (diabetic lipid control, $86.1 \%$ vs. $88.1 \%$, as well as influenza vaccine $\geq 65$ years, $78.1 \%$ vs. $80.1 \%$ ) to $7.3 \%$ (PTSD screening follow-up, $78.1 \%$ vs. $85.4 \%$ ). For the final of the 16 metrics (timely follow-up after positive depression screening, $n=2209$ ), limited variation in the outcome across covariate levels precluded analysis and the metric was dropped. For more complex patients (those with $>5$ chronic diseases), greater PACT implementation was significantly associated with higher quality for only 2 of 15 metrics (diabetic glycemic control, $P<0.001$; IHD lipid control, $P=0.02$ ).

There was no difference in effect sizes across comparable categories of PACT implementation (i.e., no effect modification) for patients with a prior hospitalization in 2012 (Supplemental Table e1), nor a difference by total primary care visits in 2012. Including only clinics with $\geq 5$ patients resulted in a similar pattern and effect sizes in quality metrics as the primary analysis, although an additional 2 metrics reached significance: influenza vaccination $18-64$ years $(P=0.03)$ and timely follow-up after positive alcohol misuse screening $(P<0.01$, Supplemental Tables e2-3).

\section{DISCUSSION}

Within a large cohort of multimorbid patients receiving care from VA primary care PACT teams from 2012 to 2014, we found that greater PACT implementation was associated with higher quality of care for 1 in 3 metrics. For more complex patients with $\geq 5$ diseases, this association was less robust. This relationship was unchanged by severity of illness as measured by prior hospitalizations or by primary care visit frequency, and was relatively stable when low-volume clinics were excluded.

The link between medical homes and higher care quality is supported by other studies, ${ }^{12,20,27-29}$ but ours is among the 


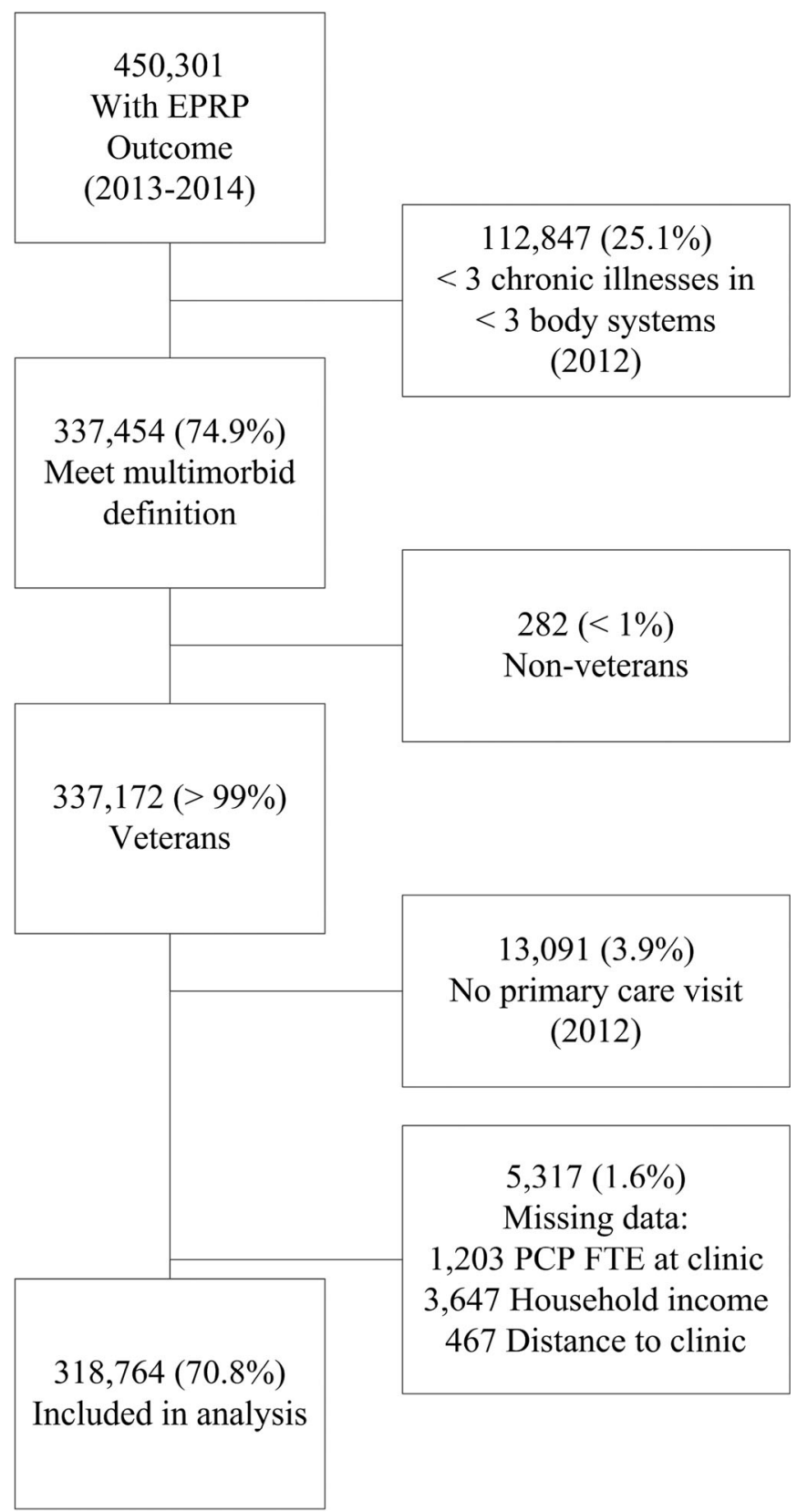

Fig. 1 Patients included in cohort of multimorbid Veterans engaged in primary care in 2012 with quality metrics measured in 2013-2014

first to examine this relationship for multimorbid patients within a large, integrated system. In prior observational work within VA primary care clinics for all patients regardless of disease burden, greater PACT implementation was associated with higher care quality for almost $40 \%$ of selected metrics. ${ }^{13}$ While fewer metrics were associated with PACT implementation in our multimorbid population, we did find significant associations for diabetic glycemic and lipid control that persisted even among high-complexity patients. This potential signal of lower quality care among multimorbid patients may reflect the "competing demands" that can occur for this population at the expense of longitudinal and preventative care, ${ }^{9}$ particularly for relatively "low-urgency" metrics like screenings. Those quality metrics representing longer-term care processes, such as glycemic or lipid control, require a higher degree of patient self-management and engagement-suggesting mechanisms wherein high performing PACTs could influence care quality if successfully engaging multimorbid patients over time. ${ }^{30,31}$

For high-complexity patients, the PACT model may be insufficient to meet their extensive care needs. We found greater complexity (i.e., disease burden) was associated with reduced probability of meeting quality metrics. This is not surprising - prior studies show that even when services are intensified for complex patients, quality outcomes remain mixed, underscoring that even well-implemented PACTs may not be sufficient to deliver high-quality care for these patients. $^{32,33}$ The relationship between complexity and care 
Table 1 Clinic and patient characteristics, overall and by quintile of clinic performance by Patient-Aligned Care Team Implementation Progress Index $\left(\mathrm{PI}^{2}\right)$ score, 2012

\begin{tabular}{|c|c|c|c|c|}
\hline & Overall, mean (SD) & Bottom category $\mathrm{PI}^{2}$ & Top category $\mathbf{P I}^{2}$ & SMD \\
\hline Patients, no. & 318,764 & 25,068 & 18,133 & - \\
\hline Age, years & $64.8(12.9)$ & $63.8(12.6)$ & $65.4(12.8)$ & 0.1 \\
\hline Female, no. $(\%)$ & $39,449(12.4)$ & 3389 (13.5) & $2196(12.1)$ & 6.2 \\
\hline Race/ethnicity, no. (\%) & & & & - \\
\hline Non-Hispanic White & $237,141(74.4)$ & $17,079(68.1)$ & $14,525(80.1)$ & 40.5 \\
\hline Non-Hispanic Black & $52,738(16.5)$ & $5550(22.1)$ & $2215(12.2)$ & 38.9 \\
\hline Hispanic & $16,068(5.0)$ & $1206(4.8)$ & $706(3.9)$ & 6.5 \\
\hline Other & $12,167(3.8)$ & $1182(4.7)$ & $659(3.6)$ & 8.1 \\
\hline Married, no. (\%) & $166,172(52.1)$ & $13,262(52.9)$ & $9623(53.1)$ & 0.5 \\
\hline Copay non-exempt, no. (\%) & $22,512(7.1)$ & $1377(5.5)$ & $1545(8.5)$ & 17.5 \\
\hline Median household income by county, $1 \mathrm{k}$ USD & $49.1(12.1)$ & $46.1(10.2)$ & $49.2(11.8)$ & 0.3 \\
\hline Distance to clinic, miles & $45.0(294.4)$ & $54.3(335.0)$ & $37.8(274.8)$ & 0.1 \\
\hline Total chronic diagnoses ${ }^{*}$ & $5.1(1.7)$ & $4.9(1.6)$ & $5.1(1.7)$ & 0.1 \\
\hline Hypertension, no. (\%) & $231,197(72.5)$ & $18,436(73.5)$ & $13,160(72.6)$ & 3.2 \\
\hline Diabetes, no. $(\%)$ & $134,118(42.1)$ & $10,615(42.3)$ & $7728(42.6)$ & 0.8 \\
\hline Depression, no. $(\%)$ & $131,297(41.2)$ & $10,843(43.3)$ & $7344(40.5)$ & 8.2 \\
\hline Ischemic heart disease, no. (\%) & $87,760(27.5)$ & $6342(25.3)$ & $5193(28.6)$ & 11.1 \\
\hline Alcohol use disorder, no. (\%) & $35,780(11.2)$ & $2542(10.1)$ & $2062(11.4)$ & 5.8 \\
\hline Chronic heart failure, no. (\%) & $33,145(10.4)$ & $2418(9.7)$ & $1798(9.9)$ & 1.3 \\
\hline Clinic visits ${ }^{*}$ & & & & - \\
\hline Primary care & $5.8(5.3)$ & $5.7(5.1)$ & $6.1(5.4)$ & 0.1 \\
\hline Specialty care & $2.0(3.9)$ & $1.8(3.5)$ & $2.0(3.6)$ & 0.1 \\
\hline Mental health & $6.0(16.7)$ & $5.1(14.0)$ & $6.0(17.2)$ & 0.1 \\
\hline All-cause VA hospitalizations ${ }^{*}$ & $0.3(1.0)$ & $0.3(0.9)$ & $0.3(0.9)$ & 0.0 \\
\hline Clinics, total ${ }^{*}$ & 917 & 87 & 83 & - \\
\hline Patients seen in rural clinic, no. (\%) & $61,491(19.2)$ & $6192(24.7)$ & $7456(41.1)$ & 52.1 \\
\hline Patients seen in CBOC, no. $(\%)^{t}$ & $148,164(46.5)$ & $12,799(51.1)$ & $11,341(62.5)$ & 41.8 \\
\hline Primary care providers per $10 \mathrm{k}$ patients & $13.1(1.9)$ & $12.8(1.5)$ & $13.4(1.7)$ & 0.4 \\
\hline
\end{tabular}

Mean (SD), except where otherwise indicated. SMD, standardized mean difference as bias-corrected effect size.

* Not included as covariate in models.

fCommunity-based outpatient clinic

quality is nuanced. Previously established relationships between disease groupings and care quality ${ }^{7,34}$ show that quality of care may be contingent on which disease combinations occur among patients. ${ }^{35}$ Patients and PCPs may also not agree about care priorities in the presence of higher disease burdens or non-illness-related demands, ${ }^{36}$ which in turn could affect achievement of longitudinal care goals and related quality metrics. Finally, while EPRP sampling is restricted to those with sufficient life expectancy, some metrics may still inadequately exclude frail and complex patients, lowering validity

Table 2 Predicted probability (95\% confidence interval) of meeting quality metric for patients seen in primary care clinics, by category of Patient-Aligned Care Team Progress Implementation Index ( $\left.\mathrm{PI}^{2}\right)$

\begin{tabular}{|c|c|c|c|c|c|c|c|}
\hline Metric & $N$ & $\mathrm{PI}^{2}-5$ to -7 & $P I^{2}-4$ to -2 & $P I^{2}-1$ to 1 & $\mathrm{PI}^{2} 2$ to 4 & $\mathrm{PI}^{2} 5$ to 8 & $P^{*}$ \\
\hline $\mathrm{CHF} \mathrm{ACE}^{\dagger}$ & 3486 & 89 (85-93) & $88(85-91)$ & $87(86-89)$ & $89(86-92)$ & $84(78-90)$ & 0.49 \\
\hline \multicolumn{8}{|l|}{ Diabetes } \\
\hline $\mathrm{A}_{1} \mathrm{C}<9 \%$ & 59,852 & $80(78-81)$ & $81(80-82)$ & $82(82-83)$ & $82(81-83)$ & $83(81-84)$ & $<0.001$ \\
\hline Foot exam & 67,320 & $88(85-90)$ & $87(86-88)$ & $88(88-89)$ & $88(86-89)$ & $90(88-92)$ & 0.10 \\
\hline LDL $<100$ & 59,826 & $86(85-87)$ & $87(86-88)$ & $88(87-88)$ & $88(87-89)$ & $88(87-89)$ & 0.04 \\
\hline $\mathrm{BP}<140 / 90$ & 59,852 & $78(77-80)$ & $78(77-79)$ & $79(79-80)$ & $79(78-80)$ & $80(78-81)$ & 0.34 \\
\hline $\mathrm{IHD}^{-} \mathrm{LDL}^{\ddagger}$ & 40,202 & $69(67-71)$ & $70(68-71)$ & $71(70-72)$ & $72(71-74)$ & $72(70-74)$ & $<0.01$ \\
\hline $\mathrm{HTN}<140 / 90^{\S}$ & 126,364 & $77(76-78)$ & $77(76-78)$ & $77(77-78)$ & $78(77-79)$ & $79(77-80)$ & 0.32 \\
\hline \multicolumn{8}{|l|}{ Vaccines } \\
\hline 18-64 years flu & 27,481 & $64(62-66)$ & $64(63-66)$ & $64(63-65)$ & $67(65-68)$ & $66(63-68)$ & 0.05 \\
\hline$>6$ years flu & 43,508 & $78(76-80)$ & $78(77-79)$ & $77(76-78)$ & $79(78-80)$ & $80(78-82)$ & 0.02 \\
\hline$\ulcorner 65$ years PPSV23 & 79,102 & $93(92-94)$ & $93(93-94)$ & $93(93-94)$ & $94(93-94)$ & $93(92-94)$ & 0.06 \\
\hline \multicolumn{8}{|l|}{ ATcohol use } \\
\hline Screening & 185,447 & $64(62-66)$ & $64(63-66)$ & $64(63-65)$ & $67(65-68)$ & $66(63-68)$ & 0.19 \\
\hline Screen $f / u^{\| l}$ & 7946 & $78(76-80)$ & $78(77-79)$ & $77(76-78)$ & $79(78-80)$ & $80(78-82)$ & 0.06 \\
\hline \multicolumn{8}{|l|}{ Depression } \\
\hline Screening & 115,002 & $96(96-97)$ & $96(96-97)$ & $96(96-96)$ & $96(96-97)$ & $97(96-97)$ & 0.23 \\
\hline \multicolumn{8}{|l|}{ PTSD } \\
\hline Screening & 141,274 & 99 (99-99) & 99 (98-99) & 99 (99-99) & 99 (99-99) & 99 (99-99) & 0.74 \\
\hline Screen $\mathrm{f} / \mathrm{u}^{\| l}$ & 3213 & $78(72-84)$ & $85(82-88)$ & $85(83-91)$ & $88(84-91)$ & $85(80-91)$ & 0.02 \\
\hline
\end{tabular}

Models adjusted for patient characteristics (age, sex, racelethnicity, copay exemption, marital status, median household income by county, and travel distance to clinic), and facility characteristics (full time providers/10,000 patients, rural or urban location, and hospital or community-based affiliation). $* P$ value for trend across categories of $P^{2}$, in ANOVA. ${ }^{\dagger}$ Congestive heart failure patients with left ventricular ejection fraction $<40 \%$, on ACE inhibitor or ARB. Ischemic heart disease patients with low-density lipoprotein under 100 or on moderate-dose statin. ${ }^{\mathcal{S}}$ Hypertensive patients with blood pressure $\leq 140 / 90 \mathrm{mmHg}$. "Positive screening with follow-up disposition in $24 \mathrm{~h}$ 
of these measures as a way to capture quality of care. ${ }^{37}$ These factors underscore that for complex patients, some of the benefits of the PCMH may be better represented by universal outcomes such as health-related quality of life. ${ }^{14,38,39}$

Our study has limitations. First, we used administrative data for defining multimorbidity. Diagnosis codes not reflecting true pathology differences between patients could lead to difficulty distinguishing multimorbid and general PACT patients. ${ }^{40}$ However, our findings were consistent as our specificity of multimorbidity definition increased, and there is little impetus to "up-code" diagnoses within integrated systems. Secondly, we note some baseline differences in patients receiving care from high and low PACT implementation clinics. Despite attempts to control for confounding, unobserved factors may influence our findings. Additionally, as we did not correct for multiple outcomes given lack of expectation of a type I versus type II error, we recognize the possibility that some of our findings may be due to statistical chance. ${ }^{41,42}$ Our study was also limited to VA data. As those with the greatest medical needs are more likely to use services under both VA and Medicare, ${ }^{43,44}$ our subgroup findings could be explained by high-complexity patients receiving care outside the VA. We did not assess the influence that disease "concordance" or similarity may have on the quality of care. Although we attempted to capture a definition of multimorbidity greater than simple disease counts by including a requirement of crossing body systems, the degree to which diagnoses share common treatment goals may influence measures of quality. ${ }^{45}$

In conclusion, we found that greater implementation of the medical home model within a large integrated health system was associated with higher probability of meeting several quality metrics for a national cohort of multimorbid patients. This finding was tempered by patient disease burden, but not prior utilization. This adds to the evidence of the overall benefits of the PCMH model, but suggests that patient complexity may be an important arbiter of care quality as measured by current quality metrics. Finally, our findings emphasize the need to capture the more meaningful measures of care quality for complex multimorbid patients.

Corresponding Author: Linnaea Schuttner, MD, MS; Health Services Research \& Development, VA Puget Sound Health Care System, 1660 South Columbian Way, Seattle, WA 98108, USA (e-mail: linnaea. schuttner@va.gov).

Funding Information This work was undertaken as part of the PACT Demonstration Laboratory initiative funded by the VA Office of Patient Care Services. The primary author was supported by grant number K12HS026369 from the Agency for Healthcare Research and Quality. ES Wong was supported by a VA HSR\&D Career Development Award (No. 13-024).

\section{Compliance with Ethical Standards:}

Conflict of Interest: The authors have no additional conflicts of interest, financial or otherwise, to disclose.
Disclaimer: The content is solely the responsibility of the authors and does not necessarily represent the official views of the Department of Veterans Affairs or the Agency for Healthcare Research and Quality.

\section{REFERENCES}

1. Fortin M, Stewart M, Poitras M-E, Almirall J, Maddocks H. A systematic review of prevalence studies on multimorbidity: toward a more uniform methodology. Ann Fam Med. 2012;10(2):142-151. doi:https://doi.org/10.1370/afm. 1337

2. Barnett $\mathbf{K}$, Mercer SW, Norbury $\mathbf{M}$, Watt G, Wyke S, Guthrie B. Epidemiology of multimorbidity and implications for health care, research, and medical education: a cross-sectional study. The Lancet. 2012;380(9836):37-43. doi:https://doi.org/10.1016/S0140-6736(12) 60240-2

3. Nunes BP, Flores TR, Mielke GI, Thumé E, Facchini LA. Multimorbidity and mortality in older adults: A systematic review and metaanalysis. Arch Gerontol Geriatr. 2016;67:130-138. doi:https://doi.org/10. 1016/j.archger.2016.07.008

4. Zulman DM, Chee CP, Wagner TH, et al. Multimorbidity and healthcare utilisation among high-cost patients in the US Veterans Affairs Health Care System. BMJ Open. 2015;5(4):e007771. doi:https://doi.org/10. 1136/bmjopen-2015-007771

5. Cassell A, Edwards D, Harshfield A, et al. The epidemiology of multimorbidity in primary care: a retrospective cohort study. Br J Gen Pract. 2018;68(669):e245-e251. doi:https://doi.org/10.3399/bjgp18X695465

6. Wong ES, Rosland A-M, Fihn SD, Nelson KM. Patient-centered medical home implementation in the Veterans Health Administration and primary care use: differences by patient comorbidity burden. $J$ Gen Intern Med 2016;31(12):1467-1474. doi:https://doi.org/10.1007/s11606-0163833-9

7. Higashi T, Wenger NS, Adams $\mathbf{J L}$, et al. Relationship between number of medical conditions and quality of care. $N$ Engl J Med. 2007;356(24):24962504. doi:https://doi.org/10.1056/NEJMsa066253

8. Zullig LL, Whitson HE, Hastings SN, et al. A systematic review of conceptual frameworks of medical complexity and new model development. J Gen Intern Med. 2016;31(3):329-337. doi:https://doi.org/10. 1007/s11606-015-3512-2

9. Parchman ML, Pugh JA, Romero RL, Bowers KW. Competing demands or clinical inertia: the case of elevated glycosylated hemoglobin. Ann Fam Med. 2007;5(3):196-201. doi:https://doi.org/10.1370/afm.679

10. Wagner EH. Chronic disease management: what will it take to improve care for chronic illness? Eff Clin Pract ECP. 1998;1(1):2-4.

11. Rosland A-M, Nelson $\mathbf{K}$, Sun $\mathbf{H}$, et al. The patient-centered medical home in the Veterans Health Administration. Am $J$ Manag Care. 2013;19(7):e263-272.

12. Nelson K, Sylling PW, Taylor L, Rose D, Mori A, Fihn SD. Clinical quality and the patient-centered medical home. JAMA Intern Med. 2017;177(7):1042. doi:https://doi.org/10.1001/jamainternmed.2017. 0963

13. Nelson KM, Helfrich $\mathbf{C}$, Sun $\mathbf{H}$, et al. Implementation of the patientcentered medical home in the Veterans Health Administration: associations with patient satisfaction, quality of care, staff burnout, and hospital and emergency department use. JAMA Intern Med. 2014;174(8):1350. doi:https://doi.org/10.1001/jamainternmed.2014.2488

14. Boyd CM, Darer J, Boult C, Fried LP, Boult L, Wu AW. Clinical practice guidelines and quality of care for older patients with multiple comorbid diseases: implications for pay for performance. JAMA. 2005;294(6):716724. doi:https://doi.org/10.1001/jama.294.6.716

15. Hughes LD, McMurdo MET, Guthrie B. Guidelines for people not for diseases: the challenges of applying UK clinical guidelines to people with multimorbidity. Age Ageing. 2013;42(1):62-69. doi:https://doi.org/10. 1093/ageing/afs 100

16. Coventry $\mathbf{P}$, Lovell $\mathbf{K}$, Dickens $\mathbf{C}$, et al. Integrated primary care for patients with mental and physical multimorbidity: cluster randomised controlled trial of collaborative care for patients with depression comorbid with diabetes or cardiovascular disease. BMJ. 2015;350:h638.

17. Rogers H. Veterans Access, Choice, and Accountability Act of 2014.; 2013. https://www.congress.gov/bill/113th-congress/house-bill/3230.

18. U.S. Department of Veterans Affairs. 172VA10P2: VHA Corporate Data Warehouse - VA. 79 FR 4377. September 2014. 
19. HCUP-US Tools \& Software Page. https://www.hcup-us.ahrq.gov/toolssoftware/chronic/chronic.jsp\#download. Accessed December 14, 2017.

20. Rosland A-M, Wong E, Maciejewski M, et al. Patient-centered medical home implementation and improved chronic disease quality: a longitudinal observational study. Health Serv Res. November 2017. doi:https:// doi.org/10.1111/1475-6773.12805

21. Yoon J, Chow A. Comparing chronic condition rates using ICD-9 and ICD-10 in VA patients FY2014-2016. BMC Health Serv Res. 2017;17. doi:https://doi.org/10.1186/s12913-017-2504-9

22. Goulet JL, Erdos J, Kancir S, et al. Measuring performance directly using the Veterans Health Administration electronic medical record: a comparison with external peer review. Med Care. 2007;45(1):73-79. doi:https://doi.org/10.1097/01.mlr.0000244510.09001.e5

23. Payne RA, Abel GA, Guthrie B, Mercer sw. The effect of physical multimorbidity, mental health conditions and socioeconomic deprivation on unplanned admissions to hospital: a retrospective cohort study. Can Med Assoc J. 2013;185(5):E221-E228. doi:https://doi.org/10.1503/ cmaj. 121349

24. Bayliss EA, Ellis JL, Shoup JA, Zeng C, McQuillan DB, Steiner JF Association of patient-centered outcomes with patient-reported and ICD9-based morbidity measures. Ann Fam Med. 2012;10(2):126-133. doi:https://doi.org/10.1370/afm.1364

25. Bayliss EA, Bayliss MS, Ware JE, Steiner JF. Predicting declines in physical function in persons with multiple chronic medical conditions: what we can learn from the medical problem list. Health Qual Life Outcomes. 2004;2:47. doi:https://doi.org/10.1186/1477-7525-2-47

26. Tonelli M, Wiebe $\mathbf{N}$, Manns BJ, et al. Comparison of the complexity of patients seen by different medical subspecialists in a universal health care system. JAMA Netw Open. 2018;1(7):e184852. doi:https://doi.org/ $10.1001 /$ jamanetworkopen.2018.4852

27. Maeng DD, Graf TR, Davis DE, Tomcavage J, Bloom FJ. Can a patientcentered medical home lead to better patient outcomes? The quality implications of Geisinger's ProvenHealth Navigator. Am J Med Qual. 2012;27(3):210-216. doi:https://doi.org/10.1177/1062860611417421

28. Solberg LI, Asche SE, Fontaine P, Flottemesch TJ, Anderson LH Trends in quality during medical home transformation. Ann Fam Med. 2011;9(6):515-521. doi:https://doi.org/10.1370/afm.1296

29. Swietek KE, Domino ME, Beadles C, et al. Do medical homes improve quality of care for persons with multiple chronic conditions? Health Serv Res. 2018;53(6):4667-4681. doi:https://doi.org/10.1111/1475-6773. 13024

30. Lauffenburger JC, Shrank WH, Bitton A, et al. Association between patient-centered medical homes and adherence to chronic disease medications: a cohort study. Ann Intern Med. 2017;166(2):81-88. doi:https://doi.org/10.7326/M15-2659

31. Beadles CA, Farley JF, Ellis AR, et al. Do medical homes increase medication adherence for persons with multiple chronic conditions?: Med Care. 2015;53(2):168-176. doi:https://doi.org/10.1097/MLR. 0000000000000292

32. Smith SM, Wallace E, O'Dowd T, Fortin M. Interventions for improving outcomes in patients with multimorbidity in primary care and community settings. Cochrane Database Syst Rev. 2016;3:CD006560. doi:https://doi.org/10.1002/14651858.CD006560.pub3
33. Yoon J, Chang E, Rubenstein LV, et al. Impact of primary care intensive management on high-risk Veterans' costs and utilization: a randomized quality improvement trial. Ann Intern Med. June 2018. doi:https://doi. org/10.7326/M17-3039

34. Woodard LD, Urech T, Landrum CR, Wang D, Petersen LA. Impact of comorbidity type on measures of quality for diabetes care. Med Care. 2011;49(6):605-610. doi:https://doi.org/10.1097/MLR. Ob013e31820f0ed0

35. Zulman DM, Asch SM, Martins SB, Kerr EA, Hoffman BB, Goldstein MK. Quality of care for patients with multiple chronic conditions: the role of comorbidity interrelatedness. J Gen Intern Med. 2014;29(3):529-537. doi:https://doi.org/10.1007/s11606-013-2616-9

36. Zulman DM, Kerr EA, Hofer TP, Heisler M, Zikmund-Fisher BJ. Patient-provider concordance in the prioritization of health conditions among hypertensive diabetes patients. J Gen Intern Med. 2010;25(5):408414. doi:https://doi.org/10.1007/s11606-009-1232-1

37. MacLean CH, Kerr EA, Gaseem A. Time out - Charting a path for improving performance measurement. $N$ Engl J Med. 2018;378(19): 17571761. doi:https://doi.org/10.1056/NEJMp1802595

38. Schuttner L, Reddy A, Rosland A-M, Nelson K, Wong ES. Association of the implementation of the patient-centered medical home with quality of life in patients with multimorbidity. $J$ Gen Intern Med. In Press.

39. Tinetti ME, Fried TR, Boyd CM. Designing health care for the most common chronic condition-multimorbidity. JAMA. 2012;307(23):24932494. doi:https://doi.org/10.1001/jama.2012.5265

40. Thomas BS, Jafarzadeh SR, Warren DK, McCormick S, Fraser VJ, Marschall $\mathbf{J}$. Temporal trends in the systemic inflammatory response syndrome, sepsis, and medical coding of sepsis. BMC Anesthesiol. 2015;15:169. doi:https://doi.org/10.1186/s12871-015-0148-Z

41. Rothman K. No adjustments are needed for multiple comparisons. Epidemiology. 1990;1(1):43-46.

42. Feise RJ. Do multiple outcome measures require p-value adjustment? BMC Med Res Methodol. 2002;2(1). doi:https://doi.org/10.1186/14712288-2-8

43. Hynes DM, Koelling $\mathbf{K}$, Stroupe $\mathbf{K}$, et al. Veterans' access to and use of Medicare and Veterans Affairs Health care. Med Care. 2007;45(3):214223.

44. Humensky J, Carretta H, de Groot K, Brown MM, Tarlov E, Hynes DM. Service utilization of Veterans dually eligible for VA and Medicare fee-forservice: 1999-2004. Medicare Medicaid Res Rev. 2012;2(3). doi:https:// doi.org/10.5600/mmrr.002.03.a06

45. Valderas JM, Gangannagaripalli J, Nolte E, et al. Quality of care assessment for people with multimorbidity. J Intern Med. February 2019:joim.12881. doi:https://doi.org/10.1111/joim.12881

Publisher's Note Springer Nature remains neutral with regard to jurisdictional claims in published maps and institutional affiliations. 